\title{
Efectos del entrenamiento de intervalos de alta intensidad en altitud simulada. Revisión sistemática
}

\section{Effects of high-intensity interval training at simulated Altitude. Systematic review}

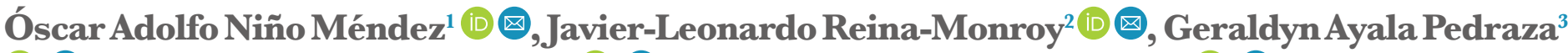 (iD $\otimes$, José-Germán Portilla-Melo ${ }^{3}$ (D) $\otimes$, Israel-David Aguilar-Romero ${ }^{3}$ (D) $\otimes$, Gristian Andrés Núñez-Espinosa ${ }^{4}$ (D) $\otimes$,Jorge-Leonardo Rodríguez-Mora ${ }^{1}$ (D) $\otimes$ \\ ${ }^{1}$ Centro de Investigación en Actividad Física, Ejercicio y Deporte (CAFED); Facultad de Ciencias del Deporte y la Educación Física; Universidad de Cundinamarca; Fusagasugá; Colombia.}

${ }^{2}$ Licenciatura en Educación Física, Recreación y Deporte UVD; Facultad de Educación; Corporación Universitaria Minuto de Dios; Bogotá; Colombia.

${ }^{3}$ Programa Cultura Fisica y Deporte; Facultad Ciencias Pedagógicas, Humanas y Sociales; Universidad INCCA de Colombia; Bogotá; Colombia.

${ }^{4}$ Escuela de Medicina; Universidad de Magallanes; Punta Arenas; Chile.

\section{Correspondencia}

Oscar Adolfo Niño Méndez, email:

oanino@ucundinamarca.edu.co

\section{Citar así \\ Niño Méndez, Óscar Adolfo; Reina-Monroy, Javier-Leonardo; Ayala Pedraza, Geraldyn; Portilla-Melo, José-Germán; Aguilar-Romero, Israel-David; Núñez-Espinosa, Cristian Andrés; Rodríguez-Mora, Jorge-Leonardo. (2021). Efectos del entrenamiento de intervalos de alta intensidad en altitud simulada. Revisión sistemática. Revista de Investigación e Innovación en Ciencias de la Salud. 3(1), 98-115. https://doi. org/10.46634/riics.50}

Recibido: 19/11/2020

Revisado: $01 / 03 / 2021$

Aceptado: 12/03/2021

\section{Editor}

Jorge Mauricio Cuartas Arias, Ph.D.

\section{Coeditor}

Fraidy-Alonso Alzate-Pamplona, MSc. ic

\section{Resumen}

Introducción: en la actualidad, los entrenadores buscan la manera de mejorar las capacidades físicas de los atletas mediante diferentes estrategias de entrenamiento, como la exposición constante o intermitente a la altitud y el entrenamiento de intervalos de alta intensidad.

Objetivo: Revisar la literatura actual y describir los efectos sobre el organismo del entrenamiento de intervalos de alta intensidad en altitud simulada en sujetos sedentarios, físicamente activos y entrenados.

Resultados: el número de artículos revisados evidencia que, en hipoxia simulada en cámara hipobárica o normobárica $(\mathrm{n}=13)$ o máscara de simulación de altitud $(\mathrm{n}=1)$, todos utilizaron intensidades altas $(n=13)$ a submáximas $(n=1)$. Los participantes de las investigaciones fueron mujeres con obesidad sedentarias $(n=3)$, hombres y mujeres físicamente activos $(\mathrm{n}=9)$ y sujetos entrenados $(\mathrm{n}=3)$. El tiempo de intervención de los estudios fue de 3 a 12 semanas, con una altitud simulada de 1824 a 4500 m.s.n.m. Se observaron efectos beneficiosos sobre la composición corporal, aptitud cardiorrespiratoria, aumentos en hemoglobina, eritropoyetina, consumo energético, fuerza máxima concéntrica e isométrica, fuerza absoluta y mejor tolerancia al ejercicio (percepción del esfuerzo). 
Copyright@ 2021. Fundación Universitaria María Cano. La Revista de Investigación e Innovación en Ciencias de la Salud proporciona acceso abierto a todo su contenido bajo los términos de la licencia Creative Commons Attribution-NonCommercial-NoDerivatives 4.0 International (CG BY-NG-ND 4.0).

Declaración de intereses

Los autores han declarado que no hay conflicto de intereses.

\section{Disponibilidad de datos}

Todos los datos relevantes se encuentran en el artículo. Para mayor información, comunicarse con el autor de correspondencia.

\section{Financiamiento}

Ninguno. Esta investigación no recibió ninguna subvención específica de agencias de financiamiento en los sectores público, comercial o sin fines de lucro.

\section{Descargo de responsabilidad}

El contenido de este artículo es responsabilidad exclusiva de los autores y no representa una opinión oficial de sus instituciones ni de la Revista de Investigación e Innovación en Ciencias de la Salud.
Conclusiones: La combinación de entrenamientos de intervalos de alta intensidad, combinado con una exposición en altitud simulada, puede evidenciar mejoras significativas en el rendimiento cardiorrespiratorio, así como en aspectos de composición corporal, lo que permitiría una mejor predisposición a intensidades más elevadas de actividad y ejercicio físico.

\section{Palabras Claves}

Actividad física, ejercicio, deporte, entrenamiento, altitud, alta intensidad, hipoxia, normoxia, hipobaria, normobaria.

\section{Abstract}

Introduction: Today, coaches are looking for ways to improve athletes' physical abilities through different training strategies, such as constant or intermittent exposure to altitude and high intensity interval training.

Objective: To review the current literature and describe the effects on the body of simulated high-intensity interval training at altitude in sedentary, physically active, and trained subjects.

Results: the number of articles reviewed evidences that, in simulated hypoxia in hypobaric or normobaric chamber $(\mathrm{n}=13)$ or altitude simulation mask $(\mathrm{n}=1)$, all used high intensities $(n=13)$ to submaximal $(n=1)$. The research participants were women with sedentary obesity $(\mathrm{n}=3)$, physically active men and women $(\mathrm{n}=9)$, and trained subjects $(n=3)$. The intervention time of the studies was 3 to 12 weeks, with a simulated altitude of 1824 to 4500 meters. Beneficial effects on body composition were observed, as well as cardiorespiratory fitness, increases in hemoglobin, erythropoietin, energy consumption, concentric and isometric maximum strength, absolute strength and better exercise tolerance (perception of effort).

Conclusions: The combination of high intensity interval training combined with a simulated altitude exposure can show significant improvements in cardiorespiratory performance, as well as in aspects of body composition, which would allow a better predisposition to higher intensities of activity and physical exercise.

\section{Keywords}

Physical activity, exercise, sport, training, altitude, high intensity, hypoxia, normoxia, hypobaric, normobaric.

\section{Introducción}

La actividad física, el ejercicio físico y el deporte se consideran los medios más importantes y utilizados actualmente para la prevención de enfermedades no trasmisibles (ENT), ocasionadas por el sedentarismo, el cual se entiende como un estilo de vida en donde prevalece la falta de actividad física [1]. El ejercicio físico es una actividad estructurada, planificada y dirigida al mejoramiento de la condición física de una persona y se considera como una subcategoría de la actividad física [2]. En la actualidad, se han implementado distintas estrategias y métodos de entrenamiento que buscan mejorar la condición física, la prevención y la rehabilitación de lesiones [3]. Entendiendo esto, se proponen metodologías de entrenamiento que buscan mayor eficacia y eficiencia para el desarrollo de las capacidades físicas, entre las que se en- 
cuentran el entrenamiento continuo extensivo, donde la carga tiene una duración larga, entre 30 minutos a 2 horas, a una intensidad moderada, que corresponde entre el umbral aeróbico y anaeróbico (1,5-3 mmol/L de lactato). Se ha demostrado que su aplicación al 70-75\% de la frecuencia cardiaca máxima ( $\left.\mathrm{FG}_{\text {máx }}\right)$ presenta beneficios a nivel cardiorrespiratorio, consumo máximo de oxígeno $\left(\mathrm{VO}_{2 \text { max }}\right)$, función endotelial periférica y en algunos factores de riesgo cardiovascular [4]. Teniendo en cuenta los bajos niveles de actividad física en la población actual, se ve la necesidad de diseñar métodos diferentes y atractivos que involucren a las personas a mejorar su estado físico [5], sumado a que en el entrenamiento deportivo se aplican múltiples métodos y metodologías de entrenamiento, que buscan adaptarse a las necesidades fisicas y deportivas en beneficio del rendimiento físico [6].

Es así como nace el entrenamiento de intervalos de alta intensidad (HIIT), el cual es un entrenamiento utilizado en el rendimiento deportivo y recreativo, puesto que ha tenido gran aceptación desde hace varios años. Esto se debe a su alta variedad de aplicación y sus notables resultados [7], permitiendo trabajar en distintos campos, sin perder sus características metodológicas, y acoplándose al objetivo de cada sujeto en cuanto a intensidad, volumen, densidad y carácter del ejercicio [8]. Dentro de los beneficios descritos por el entrenamiento HIIT, se encuentra que hay adaptaciones cardiovasculares en personas con complicaciones cardiacas, que ayudan al manejo de la enfermedad metabólica [9]. Por otro lado, en personas con sobrepeso u obesidad, mejora la capacidad aeróbica, el metabolismo de la glucosa y el perfil lipídico [10]. En sujetos entrenados, beneficia la aptitud aeróbica y función cardiovascular [11]. Finalmente, en personas físicamente activas y sanas actúa frente la constancia en la salud metabólica [12] y en adaptaciones sobre el componente cardiorrespiratorio [13].

Día a día, los entrenadores buscan la manera de mejorar las capacidades físicas de los atletas, mediante diferentes estrategias de entrenamiento, como la exposición constante o intermitente a la altitud [14]. El término altitud se refiere a elevaciones superiores a los 1500 metros sobre el nivel del mar (m.s.n.m). En un ambiente de altitud, la presión barométrica disminuye, generando un ambiente hipobárico, que conlleva a una menor difusión pulmonar y bajo transporte de oxígeno a los tejidos corporales; a esta disminución se le denomina hipoxia [15]. A raíz de esto, el organismo puede presentar efectos como aturdimiento, mareos, confusión mental y dificultad en la visión, al hallarse por encima de esta altitud [16].

Atendiendo a lo mencionado anteriormente, es importante realizar una revisión en la literatura actual, con el fin de dar respuesta a las incógnitas referentes a los efectos que tiene el entrenamiento HIIT en combinación con la altitud simulada. Por lo tanto, el objetivo de esta revisión fue describir los efectos del entrenamiento HIIT en altitud simulada sobre las medidas de composición corporal, aptitud cardiorrespiratoria, reacción molecular y componente muscular en sujetos sedentarios, físicamente activos y entrenados.

\section{Metodología}

\section{Estrategia de Búsqueda}

Se realizaron búsquedas en las bases de datos de NCBI, PubMed, Wolter Kluwer, Springer link, Research Gate, Redalyc, Ebsco, Dialnet, Science Direct y OVID entre los años 2010 y 2020. Con el fin de desarrollar una búsqueda de calidad, se tuvo en cuenta que las revistas estuvieran indexadas en el SJR (SCImago Journal Ranking), JCR (Journal Citation Reports el SCI o SSCI), categorizadas del Q1 hasta Q4, permitiendo identificar su incidencia de citación y prestigio. Se realizaron búsquedas manuales en la lista de referencias de estudios 
relevantes y estudios de revisión sistemática, para obtener artículos adicionales. Los términos de búsqueda fueron: High intensity interval training (HIIT), training at height, physiology effects, cardiorespiratory, sanguine y hormonal. Además, de su combinación a partir de los operadores booleanos (and, or, not), en idioma español, inglés y portugués. Como ejemplo, al utilizar la búsqueda (HIIT and Hypoxia) en la base de datos NCBI, solo teniendo en cuenta artículos publicados desde el año 2010 en adelante, se obtuvieron un total de 15 artículos. Una vez se hizo la revisión de cada uno de estos artículos, comenzando con el título, el resumen, la metodología, los resultados y las conclusiones, se determinó que solo cuatro [17-20] cumplían con las características y congruencia en relación al estudio pretendido.

\section{Criterios de Selección}

Para la inclusión de artículos se consideró que tuvieran una intervención con el método Intermitente de Alta Intensidad (HIIT) en altitud simulada $\geq 1500$ m.s.n.m. Se incluyeron al igual estudios en los cuales los participantes se encontraban entre 13 a 45 años. No hubo exclusiones por el género, patologías, tiempo de intervención, ni del diseño del estudio. No se tuvieron en cuenta los artículos que no hicieran parte de los índices mencionados anteriormente. Consecuentemente, el investigador principal seleccionó los datos, los cuales fueron confirmados con exactitud por el grupo de autores. Los resultados de búsqueda se anexaron en Mendeley, herramienta administradora de referencias, la cual permitió excluir los artículos duplicados. Se tuvieron en cuenta solo los manuscritos completos para una evaluación adicional de la elegibilidad. Finalmente, el proceso de selección se realizó a través del modelo de diagrama PRISMA (Figura 1).

\section{Resultados}

Todos los estudios se realizaron con base a diseños experimentales. En cada estudio, al menos un grupo realizó un protocolo HIIT con hipoxia simulada en cámara hipobárica o normobárica $(n=13)$ o máscara de simulación de altitud $(n=1)$. Todos utilizaron intensidades altas $(\mathrm{n}=13)$ a submáximas $(\mathrm{n}=1)$. Los participantes de las investigaciones fueron mujeres con obesidad sedentaria $(n=3)$, hombres y mujeres físicamente activos $(n=9)$ y sujetos entrenados $(\mathrm{n}=3)$. El tiempo de intervención de los estudios fue de 3 a 12 semanas, con una altitud simulada de 1824 a 4500 m.s.n.m. Las Tablas 1, 2 y 3 proporcionan una descripción individual más detallada de los estudios, que incluyen autor y año de publicación, tamaño de la muestra, edad, sexo e índice de masa corporal (IMC), protocolo llevado a cabo, hipoxia simulada y principales resultados.

En los resultados se observaron efectos beneficiosos sobre la composición corporal, aptitud

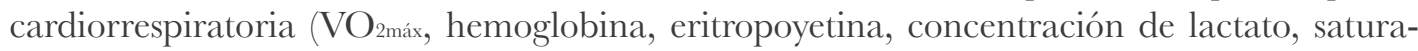
ción de oxígeno, cociente respiratorio, frecuencia cardiaca, FG máx, flujo sanguíneo cutáneo), bioquímicos (factor-1-alfa, óxido nítrico, factor de crecimiento endotelial vascular, factor de crecimiento transformable, consumo energético), fuerza (fuerza máxima concéntrica e isométrica, fuerza absoluta y relativa, potencia máxima de salida) y mejor tolerancia al ejercicio (percepción del esfuerzo). 


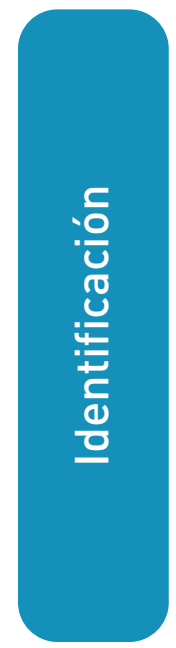
Registros identificados a través de la búsqueda en las bases de datos ( $n=1879$ )

PubMed ( $\mathrm{n}=708)$

Wolter Kluwer ( $\mathrm{n}=168$ )

Springer Link ( $\mathrm{n}=233$ )

Research Gate ( $n=135)$

Redalyc $(n=120)$

Ebsco $(n=163)$

Dialnet $(n=123)$

Science Direct $(n=121)$

Ovid ( $n=108)$

\section{Registros adicionales identificados a través de otras fuentes

$$
\text { ( } n=0)
$$

$\downarrow$

$\downarrow$
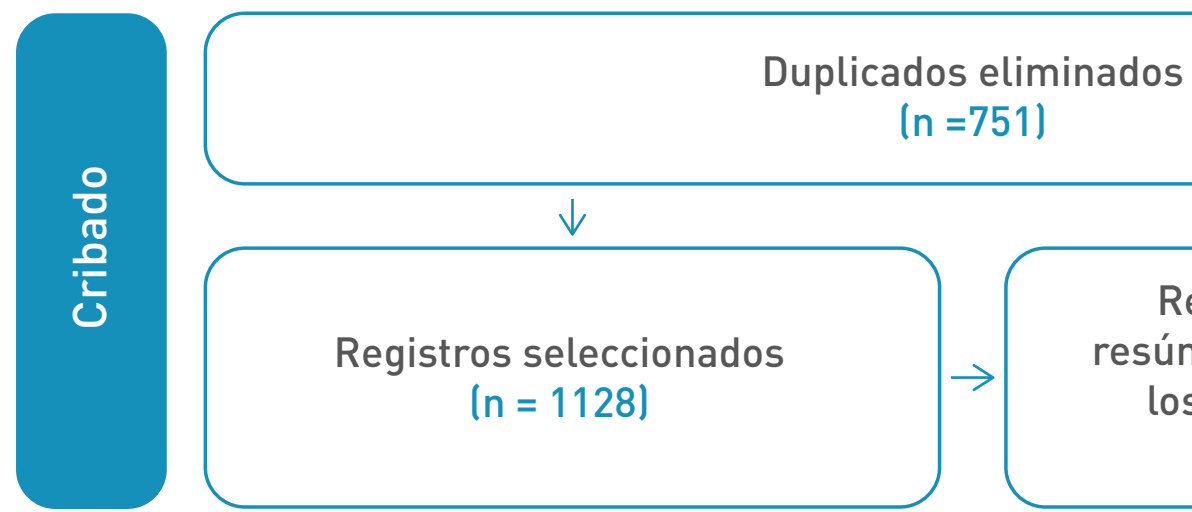

( $n=751$ )

$\downarrow$

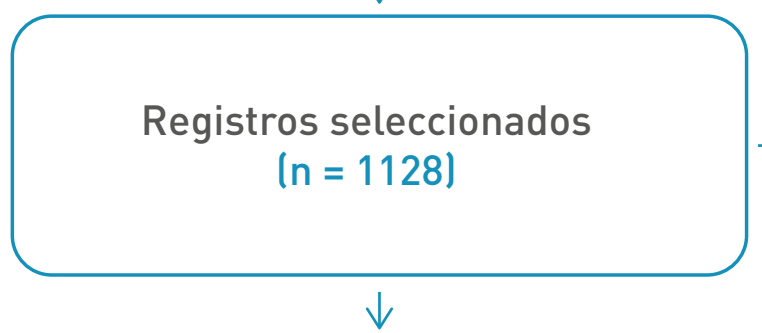

Registros excluidos por resúmenes que no cumplieron los criterios de inclusión ( $n=1079$ )
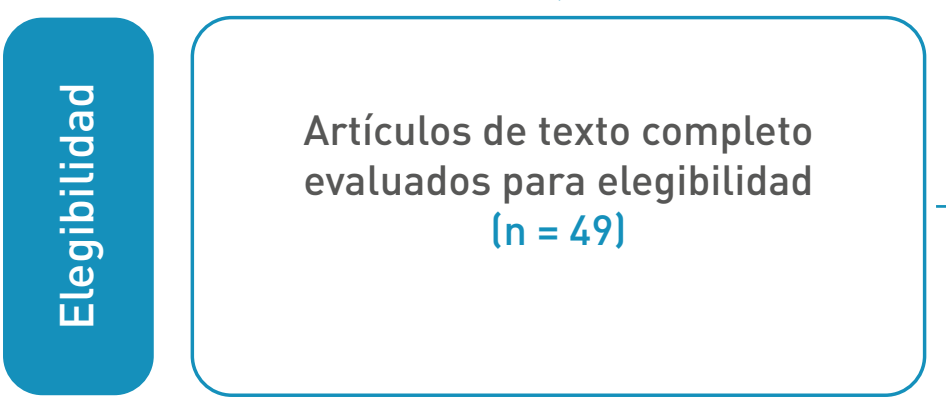

Artículos no categorizados ( $n=19$ ) Diseños no experimentales ( $n=9$ ) Congruencia con la idea de investigación ( $\mathrm{n}=7$ )

$$
\text { ( } n=35 \text { ) }
$$
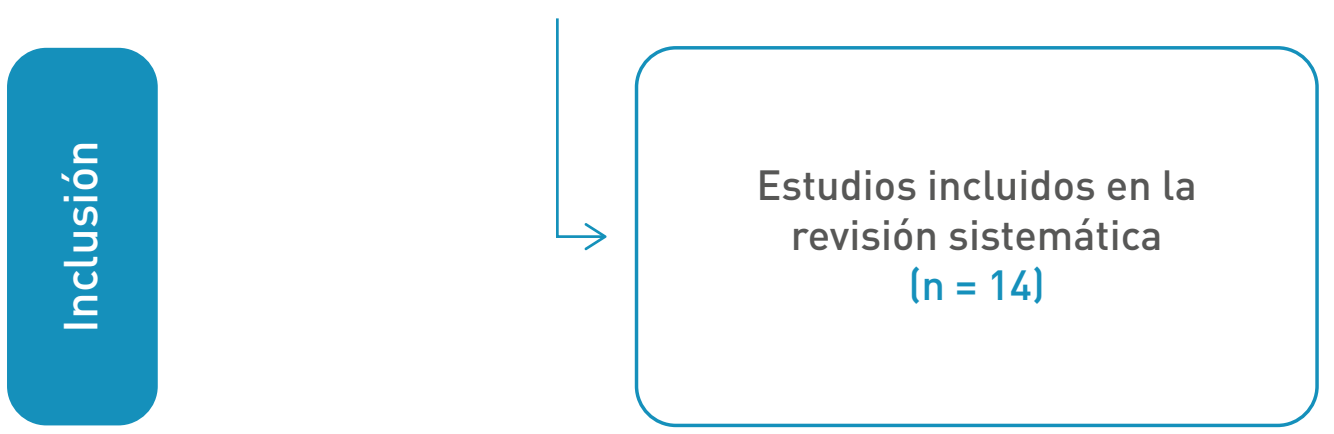

Figura 1. Diagrama para la selección de los artículos de análisis y estudios incluidos en la revisión. 


\section{Tabla 1. Sujetos Sedentarios}

\begin{tabular}{|c|c|c|c|c|c|}
\hline Referencia & $\begin{array}{l}\text { Población } \\
\text { Grupo } \\
\text { hipóxico }\end{array}$ & $\begin{array}{c}\mathrm{IMC} \\
\mathrm{kg} / \mathrm{m}^{2}\end{array}$ & Protocolo & $\begin{array}{l}\text { Exposición } \\
\text { Hipóxica } \\
\text { simulada }\end{array}$ & Resultados \\
\hline (21) & $\begin{array}{l}n=13 \\
\text { Sexo: } F \\
\text { Edad media: } \\
44,1 \pm 7,6\end{array}$ & $29,0 \pm 5,19$ & $\begin{array}{l}\text { Sesiones: } 36 \\
\text { Semanas: } 12 \\
\text { Carga: 3' } 90 \% \mathrm{~W}_{\text {máx }} \\
\text { Recuperación: activa } \\
\text { 3' } 55 \text { a } 65 \% \mathrm{~W}_{\text {máx }}\end{array}$ & $\begin{array}{l}\text { Cámara } \\
\text { de hipoxia } \\
\text { normobárica } \\
\mathrm{FiO}_{2} 17,2 \pm 0,3 \% \\
2500 \text { m.s.n.m. }\end{array}$ & $\begin{array}{l}\text { Masa corporal: disminuyó } \\
\text { IMC: disminuyó } \\
\text { Masa grasa: disminuyó } \\
\text { Masa muscular: aumentó } \\
\text { Tasa metabólica basal: aumentó } \\
\text { Cociente respiratorio: disminuyó } \\
\text { Oxidación de grasas: aumentó } \\
\text { Oxidación de carbohidratos: disminuyó }\end{array}$ \\
\hline (19) & $\begin{array}{l}\mathrm{n}=14 \\
\text { Sexo: } \mathrm{F} \\
\text { Rango de } \\
\text { edad: } 18-30\end{array}$ & $25,8 \pm 2,3$ & $\begin{array}{l}\text { Sesiones: } 20 \\
\text { Semanas: } 5 \\
\text { Carga: } 60 \text { rep de } \\
\text { esfuerzo máximo } \\
\text { de 8" } \\
\text { Recuperación: activa } \\
\text { 12" } 20 \text { a } 30 \text { rpm }\end{array}$ & $\begin{array}{l}\text { Cámara } \\
\text { de hipoxia } \\
\text { normobárica } \\
\mathrm{FiO}_{2} 15 \% \\
2500 \text { m.s.n.m. }\end{array}$ & $\begin{array}{l}\text { Composición corporal: sin cambios } \\
\text { significativos } \\
\text { Triglicéridos: disminuyó } \\
\text { Total de colesterol: sin cambios } \\
\text { significativos } \\
\text { Colesterol de lipoproteínas de alta } \\
\text { densidad: aumentó } \\
\text { Colesterol de lipoproteínas de baja } \\
\text { densidad: sin cambios significativos }\end{array}$ \\
\hline (18) & $\begin{array}{l}n=13 \\
\text { Sexo: } F \\
\text { Edad media: } \\
40,6 \pm 9,5\end{array}$ & $28,0 \pm 5,32$ & $\begin{array}{l}\text { Sesiones: } 36 \\
\text { Semanas: } 12 \\
\text { Carga: } 30 " 130 \% \mathrm{~W}_{\text {máx }} \\
\text { Recuperación: activa } \\
\text { 3' } 55 \text { a } 65 \% \mathrm{~W}_{\text {máx }}\end{array}$ & $\begin{array}{l}\text { Cámara } \\
\text { de hipoxia } \\
\text { normobárica } \\
\mathrm{FiO}_{2} 17,2 \pm 0,3 \% \\
2500 \text { m.s.n.m. }\end{array}$ & $\begin{array}{l}\text { Circunferencia de la cintura: disminuyó } \\
\text { Porcentaje de masa de grasa en el } \\
\text { tronco: disminuyó }\end{array}$ \\
\hline
\end{tabular}

Nota. Participantes grupo hipoxia (n); sexo femenino (F) masculino (m); indice de masa corporal (IMC); carga de trabajo máxima (W $W_{\text {max }}$; fracción inspirada de oxígeno (FiO2); metros sobre el nivel del mar (m.s.n.m.); repeticiones (rep); revoluciones por minuto (rpm). 


\begin{tabular}{|c|c|c|c|c|c|}
\hline Referencia & $\begin{array}{l}\text { Población } \\
\text { Grupo } \\
\text { hipóxico }\end{array}$ & $\begin{array}{c}\mathrm{IMC} \\
\mathrm{kg} / \mathrm{m}^{2}\end{array}$ & Protocolo & $\begin{array}{c}\text { Exposición } \\
\text { simulada }\end{array}$ & Resultados \\
\hline (22) & $\begin{array}{l}\mathrm{n}=6 \\
\text { Sexo: M } \\
\text { Edad Media: } \\
23,1 \pm 3,63\end{array}$ & $23,6 \pm 3,5$ & $\begin{array}{l}\text { Sesiones: } 8 \\
\text { Semanas: } 4 \\
\text { Carga: } 2 \times 5 \text { 10" sprints } \\
\text { Recuperación: pasiva 20" }\end{array}$ & $\begin{array}{l}\text { Cámara hipóxica } \\
\text { normobárica } \\
\mathrm{FiO}_{2} 14,6 \% \\
3400 \text { m.s.n.m. }\end{array}$ & $\begin{array}{l}V_{2 \text { máx }} \text { : aumentó } \\
\text { Hemoglobina: aumentó } \\
\text { Hematocrito: aumento } \\
\text { IMC: disminuyó }\end{array}$ \\
\hline (23) & $\begin{array}{l}\mathrm{n}=10 \\
\text { Sexo: M6/F4 } \\
\text { Edad media: } \\
23,1 \pm 3,6\end{array}$ & 25,0 & $\begin{array}{l}\text { Sesiones: } 12 \\
\text { Semanas: } 6 \\
\text { Carga: } 10 \text { rep 30" Salida } \\
\text { de potencia máxima } \\
\text { Recuperación: activa 90" } \\
\text { a su propio ritmo }\end{array}$ & $\begin{array}{l}\text { Cámara hipobárica } \\
\mathrm{FiO}_{2} \text { sin especificar } \\
2484 \text { m.s.n.m. }\end{array}$ & $\begin{array}{l}\mathrm{VO}_{2 m a ́ x} \text { : aumentó } \\
\mathrm{FC}_{\text {máx }} \text { : disminuyó } \\
\text { Salida de potencia máxima: } \\
\text { aumentó }\end{array}$ \\
\hline (24) & $\begin{array}{l}\mathrm{n}=7 \\
\text { Sexo: M2/F5 } \\
\text { Edad media: } \\
26 \pm 3\end{array}$ & $\begin{array}{l}21,0 \pm 2,0 \\
22,0 \pm 2,0\end{array}$ & $\begin{array}{l}\text { Sesiones: } 6 \\
\text { Semanas: } 3 \\
\text { Carga: } 4 \text { X4' 90\% FC }{ }_{\text {máx }} \\
\text { Recuperación: activa, } \\
\text { ciclismo con baja } \\
\text { resistencia }\end{array}$ & $\begin{array}{l}\text { Cámara hipóxica } \\
\text { normobárica } \\
\mathrm{FiO}_{2} 12 \% \\
4500 \text { m.s.n.m. }\end{array}$ & $\begin{array}{l}\mathrm{VO}_{2 \text { máx: }}: \text { aumentó } \\
\mathrm{FC}_{\text {máx: }}: \text { disminuyó } \\
\mathrm{W}_{\text {máx }}: \text { aumentó }\end{array}$ \\
\hline (20) & $\begin{array}{l}\mathrm{n}=10 \\
\text { Sexo: } \mathrm{M} \\
\text { Edad media: } \\
23,9 \pm 3\end{array}$ & 21,6 & $\begin{array}{l}\text { Sesiones: } 15 \\
\text { Semanas: } 5 \\
\text { Carga: Extensión de } \\
\text { rodilla 20-25\% de 1RM } \\
\text { Recuperación: pasiva 30" }\end{array}$ & $\begin{array}{l}\text { Instalación } \\
\text { hipóxica } \\
\mathrm{FiO}_{2} 12,3 \% \\
4300 \text { m.s.n.m. }\end{array}$ & $\begin{array}{l}\mathrm{VO}_{2 m a ́ x} \text { : sin cambio significativo } \\
\text { Eritropoyetina sérica: aumentó } \\
\text { Hemoglobina: aumentó } \\
\text { Repetición máxima: aumentó } \\
\text { Fuerza de contracción } \\
\text { isométrica máxima: aumentó }\end{array}$ \\
\hline (17) & $\begin{array}{l}\mathrm{n}=6 \\
\text { Sexo: } M \\
\text { Edad media: } \\
24,4 \pm 4,0\end{array}$ & $21,8 \pm 1,2$ & $\begin{array}{l}\text { Sesiones: } 9 \\
\text { Semanas: } 3 \\
\text { Carga: } 6 \text { rep de 5' 120\% } \\
\text { del umbral de lactato } \\
\text { Recuperación: pasiva 5' }\end{array}$ & $\begin{array}{l}\text { Cámaras hipóxicas } \\
\text { normobáricas } \\
\mathrm{FiO}_{2} 15,2 \% \\
\text { m.s.n.m. sin } \\
\text { especificar }\end{array}$ & $\begin{array}{l}\mathrm{VO}_{2 m a ́} \text { : aumentó } \\
\text { Factor-1-alfa: aumentó } \\
\text { Factor de crecimiento } \\
\text { endotelial vascular: } \\
\text { aumentóFactor de crecimiento: } \\
\text { aumentó } \\
\text { Hematocrito: aumentó } \\
\text { Hemoglobina: aumentó }\end{array}$ \\
\hline (25) & $\begin{array}{l}\mathrm{n}=11 \\
\text { Sexo: } \mathrm{M} \\
\text { Edad media: } \\
28 \pm 6\end{array}$ & $25,2 \pm 2,6$ & $\begin{array}{l}\text { Sesiones: } 18 \\
\text { Semanas: } 3 \\
\text { Carga: 60" de pedaleo } \\
\text { Recuperación: activa 30" } \\
20 \text { W }\end{array}$ & $\begin{array}{l}\text { Cámaras hipóxicas } \\
\text { normobáricas } \\
\mathrm{FiO}_{2} 14,5 \% \\
\text { m.s.n.m. sin } \\
\text { especificar }\end{array}$ & $\begin{array}{l}\text { Tolerancia al ejercicio: aumentć } \\
\text { Dominio de intensidad severa: } \\
\text { aumentó } \\
\text { concentración de lactato: } \\
\text { aumentó }\end{array}$ \\
\hline
\end{tabular}




\begin{tabular}{|c|c|c|c|c|c|}
\hline (26) & $\begin{array}{l}n=15 \\
\text { Sexo: } M \\
\text { Edad: } 13\end{array}$ & $18,3 \pm 2,2$ & $\begin{array}{l}\text { Sesiones: } 2 \\
\text { Semanas: } 2 \\
\text { Carga: } 4 \text { ' de carrera } 90 \text { al } \\
95 \% \text { de } F C_{\text {máx }} \\
\text { Recuperación: activa 3' } \\
60 \text { a } 70 \% \text { FC } \text { máx }\end{array}$ & $\begin{array}{l}\text { Aire acondicionado } \\
\text { Hipoxia } \\
\text { Normobárica } \\
15,3 \% \mathrm{O}_{2}\end{array}$ & $\begin{array}{l}\text { IMC: disminuyó } \\
\text { Flujo sanguíneo cutáneo: } \\
\text { aumentó } \\
\text { FC: aumentó }\end{array}$ \\
\hline (27) & $\begin{array}{l}n=12 \\
\text { Sexo: M8/F4 } \\
\text { Edad media: } \\
M 29 \pm 3,83 / \\
F 21 \pm 0,82\end{array}$ & $\begin{array}{l}M 25,9 \pm 4,15 \\
F 21,6 \pm 9,8\end{array}$ & $\begin{array}{l}\text { Sesiones: } 12 \\
\text { Semanas: } 6 \\
\text { Carga: } 10 \text { rep 30" salida } \\
\text { de potencia máxima } \\
\text { Recuperación: activa 90" } \\
25 \text { W }\end{array}$ & $\begin{array}{l}\text { Máscara de } \\
\text { simulación de } \\
\text { altitud } \\
\text { Rango 1829-3658 } \\
\text { m.s.n.m. }\end{array}$ & $\begin{array}{l}\mathrm{VO}_{2 \text { máx }} \text { : aumentó } \\
\text { Salida de potencia máxima: } \\
\text { aumentó }\end{array}$ \\
\hline
\end{tabular}

Nota. Participantes grupo hipoxia (n); sexo femenino (F) masculino (M); índice de masa corporal (IMC); carga de trabajo máxima (W $W_{\text {max }}$ ); frecuencia cardiaca $(F C)$; consumo máximo de oxígeno $\left(V_{2 m a x}\right)$; frecuencia cardiaca máxima $\left(F C_{\text {maxx }}\right.$; fracción inspirada de oxígeno $\left(F_{i} O_{2}\right)$; metros sobre el nivel del mar (m.s.n.m.); repetición máxima (1RM); series por repeticiones (x); repeticiones (rep); vatios (W).

\begin{tabular}{|c|c|c|c|c|c|}
\hline Referencia & $\begin{array}{l}\text { Población } \\
\text { Grupo } \\
\text { hipóxico }\end{array}$ & $\begin{array}{c}\text { IMC } \\
\mathrm{kg} / \mathrm{m}^{2}\end{array}$ & Protocolo & $\begin{array}{l}\text { Exposición } \\
\text { Hipóxica } \\
\text { simulada }\end{array}$ & Resultados \\
\hline (28) & $\begin{array}{l}\mathrm{n}=8 \\
\text { Sexo: } M \\
\text { Edad media: } \\
17,1 \pm 0,3\end{array}$ & $21 \pm 1,7$ & $\begin{array}{l}\text { Sesiones: } 10 \\
\text { Semanas: } 5 \\
\text { Carga: } 2-3 \times 5-6 \\
\text { carrera 15" } \\
\text { Recuperación: } \\
\text { entre repeticiones } \\
\text { pasiva 15" / entre } \\
\text { series pasiva 10' }\end{array}$ & $\begin{array}{l}\text { Cámara hipóxica } \\
\text { normobárica } \\
\mathrm{FiO}_{2} 14,3 \% \\
2900 \text { m.s.n.m. }\end{array}$ & $\begin{array}{l}\mathrm{SpO}_{2} \text { : disminuyó } \\
\text { Saltos de contra movimiento: aumentó } \\
\text { Alta intensidad de sprint repetido: } \\
\text { aumentó }\end{array}$ \\
\hline (29) & $\begin{array}{l}\mathrm{n}=8 \\
\text { Sexo: } M \\
\text { Edad media: } \\
18,6 \pm 5,3\end{array}$ & 19,4 & $\begin{array}{l}\text { Sesiones: } 6 \\
\text { Semanas: } 2 \\
\text { Carga: Carrera de } \\
\text { intervalos de } 5 \text { rep } \\
\text { 3' } 84 \text { a } 90 \% \mathrm{VO}_{2 \text { máx }} \\
\text { Recuperación: } \\
\text { pasivo 90" }\end{array}$ & $\begin{array}{l}\text { Cámara hipóxica } \\
\text { normobárica } \\
\mathrm{FiO}_{2} 15,4 \% \\
2400 \text { m.s.n.m. }\end{array}$ & $\begin{array}{l}\mathrm{VO}_{2 m a ́}: \text { sin cambios } \\
\text { Eritropoyetina: aumentó } \\
\mathrm{FC}: \text { aumentó } \\
\mathrm{SpO}_{2} \text { : disminuyó } \\
\mathrm{La} \text { concentración en bicarbonato } \mathrm{HCO}_{3} \text { : } \\
\text { disminuyo } \\
\text { Lactato: aumentó }\end{array}$ \\
\hline (30) & $\begin{array}{l}\mathrm{n}=6 \\
\text { Sexo: } \mathrm{M} \\
\text { Edad media: } \\
22 \pm 1,6\end{array}$ & $23,5 \pm 1,5$ & $\begin{array}{l}\text { Sesiones: } 9 \\
\text { Semanas: } 3 \\
\text { Carga: } 4-5 \text { rep 4' } \\
90 \% \mathrm{VO}_{2 \text { máx }} \\
\text { Recuperación: } \\
\text { activa 4' } 60 \% \mathrm{VO}_{2 \text { máx }}\end{array}$ & $\begin{array}{l}\text { Cámara hipóxica } \\
\text { normobárica } \\
\mathrm{FiO}_{2} 15,2 \% \\
2500 \text { m.s.n.m. }\end{array}$ & $\begin{array}{l}\mathrm{VO}_{2 m \text { áx }} \text { : aumentó } \\
\mathrm{IMC}_{\text {disminuyó }} \\
\text { carga de trabajo máxima absoluta y } \\
\text { relativa: aumentó }\end{array}$ \\
\hline
\end{tabular}

Nota. Participantes grupo hipoxia (n); sexo femenino (F) masculino (m); indice de masa corporal (IMC); carga de trabajo máxima (W $W_{\text {maxx }}$ ); saturación de oxígeno $\left(\mathrm{SpO}_{2}\right)$; fracción inspirada de oxígeno ( $\left.\mathrm{FiO} 2\right)$; metros sobre el nivel del mar (m.s.n.m.); series por repeticiones (x); repeticiones (rep); vatios $(W)$. 


\section{Discusión}

Este articulo pretende elaborar una revisión sobre los diferentes efectos beneficiosos que se pueden presentar en el organismo, al realizar un entrenamiento de intervalos de alta intensidad y corta duración en altitud simulada. El análisis de los resultados obtenidos reveló efectos positivos en las diferentes poblaciones revisadas. Así mismo, se pudo observar que los entrenamientos pueden variar desde las 3 semanas hasta las 12 semanas y que la simulación en altitud puede variar desde los 1824 m.s.n.m hasta los 4500 m.s.n.m y con una fracción de oxígeno inspirada desde el 12,0\% hasta los 17,2\%.

El entrenamiento en altitud utiliza dos enfoques principales en busca de aumentos en el rendimiento deportivo: "vivir bajo y entrenar alto" [31,32] y "vivir alto-entrenar bajo" [33,34], con altitudes que pueden ir desde los 2300 hasta los 5700 m.s.n.m [35]. En altitud natural, el organismo puede presentar efectos adversos como el mal de montaña (a tan solo 2500 m.s.n.m). Estos efectos suelen presentarse a las pocas horas de permanencia. Para contrarrestar estos síntomas es necesario suministrar oxígeno y, en su posibilidad, disminuir la altitud y su tiempo de permanencia [36,37]. Sin embargo, las investigaciones actuales destacan los posibles beneficios de una exposición controlada a altitud natural o simulada $[38,39]$. Además, cuando las respuestas del organismo logran contrarrestar las exigencias de estar en altitud, se pueden presentar efectos positivos como disminución de riegos metabólicos y cardiovasculares, aumentos de enzimas musculares oxidativas [40]. Ahora, acceder en ocasiones a una altitud natural que induzca cambios significativos puede representar un gran reto. Por tal motivo, se utilizan otros mecanismos que permitan simular los efectos hipobáricos y/o hipóxicos de la altitud, tales como salas de nitrógeno, cámaras de hipoxia, cámaras hipobáricas y máscaras de hipoxia $[23,27,41,42]$. Un ambiente hipóxico normobárico se produce por dilución de nitrógeno, simulando un entorno de altitud equivalente a aproximadamente 2000 a 3000 m.s.n.m [41]. La cámara hipobárica puede simular condiciones de hasta 6000 m.s.n.m., extrayendo el $\mathrm{O}_{2}$ de la cámara e introduciéndolo de nuevo con la presión parcial de oxígeno que se reduce al nivel que se desee [41]. La máscara inductora de hipoxia, a partir de su sistema de resistencia multinivel, permite simular altitudes que van desde 914 a 5486 m.s.n.m. para simular altitud [27].

El entrenamiento HIIT se caracteriza por tener estímulos relativamente cortos de actividad intensa, intercalados por períodos de descanso activos de baja intensidad $[43,44]$. En la prescripción del ejercicio para sujetos entrenados, la intensidad de intervalo se puede definir mediante la frecuencia cardiaca de reserva (FCR), la velocidad aeróbica máxima (VAM) o a través de la escala de Borg. En cualquier caso, las intensidades deben acercarse al 90\%. Además, es importante tener en cuenta la percepción subjetiva del esfuerzo. En cuanto a la duración del intervalo, se realiza en tiempo real desde los 15 y llegando hasta los 90 o incluso los 150 segundos. Lo ideal es que una vez conocido el tiempo que necesita el sujeto para alcanzar su velocidad aeróbica máxima, se estipule el tiempo del intervalo. El número de intervalos en el HIIT no es estandarizado, ya que esto depende de la intensidad del ejercicio, el estado del sujeto y el momento de la temporada [45]. La mayoría de los autores hacen énfasis en acumular

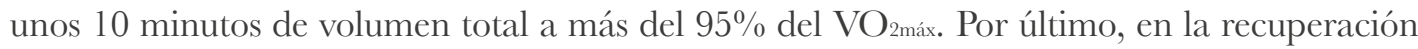
entre intervalos no existe una fórmula exacta para calcular este valor. La percepción subjetiva del esfuerzo de las personas, junto al conocimiento y experiencia del entrenador pueden servir de referentes. El tiempo de recuperación se relaciona con la duración del intervalo, aunque por norma general y, a efectos del diseño inicial de una sesión de HIIT, se puede comenzar con una relación 1:1 (puede haber recuperación activa o estática) [45]. El rendimiento de la resistencia 
aeróbica depende esencialmente de tres factores fisiológicos: economía del gesto, \% $\mathrm{VO}_{2 \text { máx }}$ sostenible durante un tiempo prolongado y la potencia aeróbica máxima. Dicho lo anterior, algunos de los objetivos del HIIT son mejorar el $\mathrm{VO}_{2 \text { máx }}$ o la potencia aeróbica, evidenciado en una mayor capacidad para realizar ejercicio en tiempo e intensidad [45].

En cuanto a las adaptaciones, se pueden nombrar a nivel fisiológico, las que están determinadas por distintos factores en los que se incluyen el modo y la naturaleza precisa del estímulo del ejercicio [7]. Se ha determinado que los protocolos de HIIT a corto plazo en personas activas sin entrenamiento mejoran la capacidad aeróbica. Así mismo, permite una remodelación fisiológica similar a los cambios inducidos por el método continuo intensivo [46]. Además, se ha descrito que solo seis sesiones de HIIT con un protocolo Wingate por dos semanas mejora la capacidad oxidativa del músculo esquelético [7]. Se debe agregar que estas son adaptaciones periféricas, al igual que el aumento del contenido mitocondrial del músculo esquelético y la densidad capilar. Dentro de las adaptaciones centrales se puede observar el aumento del $\mathrm{VO}_{2 \max }$ [47], como lo demostró un estudio de seis sesiones de entrenamiento HIIT durante dos semanas, en el que aumentó en promedio 6,1 $\pm 5,9 \%$ de su valor inicial [48]. Por otro lado, las adaptaciones del músculo esquelético regulan el metabolismo del sustrato, el cual genera mayor oxidación de grasas y mejor oxidación de carbohidratos, debido al mayor contenido mitocondrial [49]. Dicho esto, una sola sesión de HIIT activa las vías de la biogénesis mitocondrial, y es esta activación regular la que conduce al aumento de su densidad [50]. En lo que se refiere a las adaptaciones cardiovasculares, se manifiestan después de dos a cuatro semanas de comenzar un entrenamiento, generando incremento en el gasto cardiaco máximo, que se encuentra correlacionado positivamente con las adaptaciones hematológicas [51]. Se evidencia que tanto el volumen de plasma como la sangre aumentan considerablemente [52] y la frecuencia cardiaca disminuye [53].

El aumento de las publicaciones científicas enfocadas al HIIT, tanto en el ámbito del rendimiento deportivo [54] como en la salud [9], indica el valor que ha obtenido este método de entrenamiento. Es así que [55] se desarrolló un estudio con el objetivo de documentar en qué medida el HIIT y el entrenamiento de resistencia isoinercial (ERI) pueden ser efectivos para reducir los riesgos seleccionados de síndrome metabólico y enfermedades cardiometabólicas, modificar variables de composición corporal, mejorar la aptitud cardiopulmonar y la mayor capacidad para realizar ejercicio físico. El estudio aplicó durante ocho semanas un entrenamiento HIIT y ERI a 12 adultos mayores sin patologías que les impidiera el ejercicio físico. $\mathrm{Al}$ terminar el estudio, los autores concluyeron que el entrenamiento HIIT es eficaz en la mejora cardiovascular, capacidad de la aptitud y para inducir cambios positivos en cinco factores de riesgo que definen el síndrome metabólico. Además, siempre que los sujetos puedan mantener un estilo de vida activo, confirman que los factores que afectan la aptitud cardiovascular pueden no verse afectados negativamente por largos períodos de desentrenamiento. Así mismo [56], concluyeron que, tras realizar un programa de rehabilitación cardíaca en el hogar, que incluyó entrenamiento a intervalos y entrenamiento de fuerza, se asocia con una mejor capacidad aeróbica y calidad de vida para los pacientes con insuficiencia cardiaca en un grupo experimental $(n=20)$ y un grupo control $(n=20)$. El grupo experimental se sometió a un programa de entrenamiento de resistencia muscular e intervalos de alta intensidad de 12 semanas, mientras que el grupo control mantuvo sus actividades habituales de la vida diaria. Por otra parte, se han desarrollado estudios de comparación que dan confianza para aplicar este método de entrenamiento. En este caso [57], después de realizar un experimento controlado que involucró a 31 mujeres con obesidad (entre 18-30 años), las cuales fueron asignadas 
aleatoriamente a los programas de entrenamiento de cinco semanas en HIIT o entrenamiento continuo extensivo (ECE), las participantes en condición HIIT realizaron 20 minutos de ciclos repetidos de 8 segundos intercalados con intervalos de descanso de 12 segundos; aquellos en condición ECE hicieron ciclos continuos durante 40 minutos al 60-80\% VO2pico, ambos durante cuatro días a la semana, demostrando que tanto HIIT como el entrenamiento continuo extensivo son efectivos para mejorar la aptitud cardiorrespiratoria. Sin embargo, HIIT fue una estrategia más agradable y eficiente en el tiempo.

El entrenamiento deportivo aplicado en la altitud puede presentar de forma general un aumento del 14\% [58] sobre la capacidad aeróbica [40]. La respuesta de aclimatación a la altitud depende del parámetro fisiológico en cuestión, como es el caso de la correlación entre la masa de glóbulos rojos que aumenta con el tiempo cuando la presión parcial de oxígeno $\left(\mathrm{PaO}_{2}\right)$ disminuye [59]. Es por esto que la "altitud umbral" para muchas personas es de 2200 a 2500 m.s.n.m [60]. Así, la aclimatación a la hipoxia ambiental genera adaptaciones cardiorrespiratorias reflejadas en el trasporte y la utilización de oxígeno [61], aumentos en la concentración de hemoglobina en sangre, elevada capacidad de amortiguación frente a la homeostasis de elementos ácidos y básicos del organismo, beneficios sobre las propiedades estructurales y bioquímicas del músculo esquelético [61] y aumento de la ventilación pulmonar. Por otro lado, la potencia aeróbica máxima se deprime mientras los cambios en la variable hematológica compensan esta pérdida [62]. Como se mencionó anteriormente, el entrenamiento en altitud puede no ser para todas las personas, ya que esto depende inicialmente del manejo y tiempo de exposición, lo cual debe ser controlado según la edad, nivel de condición física, estado del hierro, los requisitos energéticos de cada individuo [49,63].

Continuando con lo mencionado, entre las revisiones científicas se pudo encontrar la aplicación de estas estrategias. Se ha comprobado que, a partir del entrenamiento en altura, se genera un aumento en eritropoyetina (EPO), lo que permite incrementar los valores de hemoglobina (HGB) [64], según los resultados encontrados en los atletas que participaron en su estudio, los cuales fueron: 20 atletas competitivos que se dividieron en dos grupos a partir de un diseño experimental. El primer grupo realizó una etapa de entrenamiento a una altitud de 600 m.s.n.m. (grupo control) y el segundo grupo a una altitud de 2000 m.s.n.m. (Grupo experimental). Se realizaron pruebas de laboratorio al inicio y al final para determinar los valores de EPO y HGB. Una vez realizado el análisis, se determinó que los resultados fueron: aumento del 26,5\% de la EPO en el grupo experimental y de 0,09\% en el grupo control; los valores de HGB aumentaron 4,4\% en el grupo experimental y $0,4 \%$ en el grupo control. Además, al analizar los datos se determina que al realizar una o más etapas de entrenamiento en altitud se presentan mejoras significativas sobre la VAM y el $\mathrm{VO}_{2 \text { máx }}$, concluyendo que el entrenamiento en altitud mejora significativamente el rendimiento físico en los corredores. $\mathrm{Al}$ igual, se encuentran investigaciones que demuestran que el entrenamiento en una altitud recomendada genera adaptaciones en el organismo. De esta manera, aumentando el rendimiento físico en los deportistas, tuvieron como objetivo comparar el efecto del entrenamiento hipóxico intermitente (EHI) y la estrategia (vivir alto, entrenar bajo) sobre la capacidad aeróbica y el rendimiento deportivo en ciclistas, en el cual demostraron que tras entrenar en una altura por encima de 2000 m.s.n.m, siendo deportistas ya adaptados al entrenamiento sobre el nivel del mar, se generan adaptaciones fisiológicas, que atribuyen efectos cardiovasculares, ventilatorios y adaptaciones periféricas, es decir, amortiguamiento muscular, aumento en enzimas glucolíticas y una mayor eficiencia mecánica [65]. Un año después [66], un nuevo estudio se realizó con el objetivo de evaluar la eficacia del EHI normobárico a una altura de 
2000 m.s.n.m. en la capacidad aeróbica de rendimiento físico en deportistas biatletas ( $\mathrm{n}=$ 12). Los descubrimientos de este estudio muestran mejoras significativas sobre la capacidad aeróbica en el $\mathrm{VO}_{2 \text { máx }}$ de los biatletas.

De igual forma, se ha comprobado que el EHI mejora el rendimiento anaeróbico en corredores masculinos de $400 \mathrm{~m}(\mathrm{n}=12)$, divididos en dos grupos [67]. El grupo control realizó un entrenamiento durante cuatro semanas, tres días a la semana, y el grupo experimental hizo el mismo entrenamiento en una cámara hipobárica, simulando 3000 m.s.n.m El rendimiento de carrera anaeróbica se probó con un entrenamiento de carrera de intervalos repetidos (RIR). Los sujetos realizaron tantas series como pudieron al 90\% de la velocidad máxima individual alcanzada, con un período de descanso de tres minutos entre series. Por último, con el fin de determinar si el régimen de EHI mejora la economía del ejercicio y el rendimiento del ejercicio aeróbico en nadadores moderadamente entrenados, [68], aplicaron un estudio con 20 nadadores moderadamente entrenados, asignados aleatoriamente a grupo control $(n=10)$ que hizo EHI en condiciones normóxicas y un grupo experimental $(n=10)$ que realizó EHI en altitud simulada de 3000 m.s.n.m en una cámara hipobárica. Los hallazgos indican que el EHI, en conjunto con la altitud simulada, presenta cambios significativos frente al rendimiento aeróbico en nadadores moderadamente entrenados.

\section{Conclusiones}

La combinación de entrenamientos de intervalos de alta intensidad con una exposición en altitud simulada puede evidenciar mejoras significativas en el rendimiento cardiorrespiratorio, así como en aspectos de composición corporal, lo que permitiría una mejor predisposición a intensidades más elevadas de actividad y ejercicio físico. Además, los diferentes mecanismos para simular ambientes hipobáricos e hipóxicos representarían una opción viable, cuando ir a altitud natural es un inconveniente. Sin embargo, dichos beneficios son susceptibles a las respuestas individuales de cada sujeto. Finalmente, son necesarias más investigaciones con esta combinación y con diferentes poblaciones.

\section{Referencias}

1. Crespo-Salgado JJ, Delgado-Martín JL, Blanco-Iglesias O, Aldecoa-Landesa S. Guía básica de detección del sedentarismo y recomendaciones de actividad física en atención primaria. Aten Primaria. 2015;47(3):175-83. DOI: https://doi.org/10.1016/j. aprim.2014.09.004

2. Caspersen CJ, Powell KE, Christenson GM. Physical activity, exercise, and physical fitness: definitions and distinctions for health-related research. Public Heal reports (Estados Unidos). 1985;100(2):126-31. https://www.ncbi.nlm.nih.gov/pmc/articles/PMC1424733/

3. Russell R, Michael P, Steven N, William L, Caroline A, Claude B, et al. Physical activity and public health. A recommendation from the Centers for Disease Control and Prevention and the American College of Sports Medicine. JAMA J Am Med Assoc. 1995;273(5):402-7. DOI: https://doi.org/10.1001/jama.1995.03520290054029

4. Conraads VM, Pattyn N, De Maeyer C, Beckers PJ, Coeckelberghs E, Cornelissen VA, et al. Aerobic interval training and continuous training equally improve aerobic exercise capacity in patients with coronary artery disease: The SAINTEX-CAD study. Int J Cardiol. 2015;179:203-10. DOI: https://doi.org/10.1016/j.ijcard.2014.10.155 
5. Costigan SA, Eather N, Plotnikoff RC, Taaffe DR, Lubans DR. High-intensity interval training for improving health-related fitness in adolescents: A systematic review and meta-analysis. Br J Sports Med. 2015;49(19):1253-61. DOI: http://dx.doi.org/10.1136/ bjsports-2014-094490

6. Ross LM, Porter RR, Durstine JL. High-intensity interval training (HIIT) for patients with chronic diseases. J Sport Heal Sci. 2016;5(2):139-44. DOI: https://doi.org/10.1016/j. jshs.2016.04.005

7. Gibala MJ, Jones AM. Physiological and performance adaptations to high-intensity interval training. Nestle Nutr Inst Workshop Ser. 2013;76:51-60. DOI: https://doi. org / 10.1159/000350256

8. López ChicharroJ, Vicente Campos D. HIIT de la teoría a la práctica. Madrid: Fisiología del Ejercicio; 2018.

9. Cassidy S, Thoma C, Houghton D, Trenell MI. High-intensity interval training: a review of its impact on glucose control and cardiometabolic health. Diabetol. 2017;60(1):7-23. DOI: https://doi.org/10.1007/s00125-016-4106-1

10. Kelly BM, Xenophontos S, King JA, Nimmo MA. An evaluation of low volume high-intensity intermittent training (HIIT) for health risk reduction in overweight and obese men. BMC Obesity. 2017;4:1-12. DOI: https://doi.org/10.1186/s40608-017-0151-7

11. Kilpatrick MW, Jung ME, Little JP. High-intensity interval training A Review of Physiological and Psychological Responses. ACSM's Health \& Fitness Journal. 2014;18(5):1 1-6. DOI: https://doi.org/10.1249/FIT.0000000000000067

12. Logan GRM, Harris N, Duncan S, Schofield G. A review of adolescent high-intensity interval training. Sport Med. 2014;44(8):1071-85. DOI: https://doi.org/10.1007/s40279014-0187-5

13. Wen D, Utesch T, WU J, Robertson S, Liu J, Hu G, et al. Effects of different protocols of high intensity interval training for $\mathrm{VO} 2 \mathrm{max}$ improvements in adults: A meta-analysis of randomised controlled trials. J Sci Med Sport. 2019;22(8):941-7. DOI: https://doi. org/10.1016/j.jsams.2019.01.013

14. Wolski LA, Mckenzie DC, Wenger HA. Altitude training for improvements in sea level performance. Sport Med. 1996;22(4):251-63. DOI: https://doi.org/10.2165/00007256199622040-00004

15. Wilmore JH, Costill DL. Fisiología del Esfuerzo y del Deporte. 6a Edición Revisada y Aumentada. Barcelona: Paidotribo; 2014.

16. Woodrow AD, Webb JT, Wier GS. Recollection of hypoxia symptoms between training events. Aviat Sp Environ Med. 2011;82(12):1143-7. DOI: https://doi.org/10.3357/ ASEM.2987.2011

17. Żebrowska A, Jastrzębski D, Sadowska-Krępa E, Sikora M, Di Giulio C. Comparison of the effectiveness of high-intensity interval training in hypoxia and normoxia in healthy male volunteers: A pilot study. Biomed Res Int. 2019;Article7315714. DOI: https://doi. org/10.1155/2019/7315714 
18. Camacho-Cardenosa A, Camacho-Cardenosa M, Olcina G, Timón R, Brazo-Sayavera J. Detraining effect on overweight/obese women after high-intensity interval training in hypoxia. Scand J Med Sci Sport. 2019 Apr;29(4):535-43. DOI: https://doi. org/10.1111/sms. 13380

19. Kong Z, Shi Q, Nie J, Tong TK, Song L, Yi L, et al. High-intensity interval training in normobaric hypoxia improves cardiorespiratory fitness in overweight Chinese young women. Front Physiol. 2017;8(MAR):1-11. DOI: https://doi.org/10.3389/fphys.2017.00175

20. De Smet S, van Herpt P, D'Hulst G, Van Thienen R, Van Leemputte M, Hespel P. Physiological adaptations to hypoxic vs. normoxic training during intermittent living high. Front Physiol. 2017;8(MAY):1-16. DOI: https://doi.org/10.3389/fphys.2017.00347

21. Camacho-Cardenosa A, Camacho-Cardenosa M, Burtscher M, Martínez-Guardado I, Timon R, Brazo-Sayavera J, et al. High-intensity interval training in normobaric hypoxia leads to greater body fat loss in overweight/obese women than high-intensity interval training in normoxia. Front Physiol. 2018;9(FEB):1-13. DOI: https://doi.org/10.3389/ fphys.2018.00060

22. Camacho G, Camacho Ca, Martínez G, Marcos S, Timon G. A new dose of maximal-intensity interval training in hypoxia to improve body composition and hemoglobin and hematocrit levels: a pilot study. J Sport Med Phys Fit. 2017;57(1-2):60-9. https://doi. org/10.23736/s0022-4707.16.06549-x

23. Bellovary BN, King KE, Nunez TP, Mccormick JJ, Wells AD, Bourbeau KC, et al. Effects of high-intensity interval training while using a breathing-restrictive mask compared to intermittent hypobaric hypoxia. J Hum Sport Exerc. 2019;14(4):821-33. https://doi. org/10.14198/jhse.2019.144.11

24. Menz V, Semsch M, Mosbach F, Burtscher M. Cardiorespiratory effects of one-legged high-intensity interval training in normoxia and hypoxia: A pilot study. J Sport Sci Med. 2016;15(2):208-13. https://www.ncbi.nlm.nih.gov/pmc/articles/PMC4879432/

25. Deb SK, Gough LA, Sparks SA, McNaughton LR. Sodium bicarbonate supplementation improves severe-intensity intermittent exercise under moderate acute hypoxic conditions. Eur J Appl Physiol. 2018;118(3):607-15. DOI: https://doi.org/10.1007/s00421018-3801-7

26. Suwannarat R, Srihirun K, Brito J, Suksom D. Acute Effects of Normoxic and Hypoxic High-Intensity Interval Exercise on Hemodynamics and Cutaneous Blood Flow in Youth Soccer Players. J Exerc Physiol. 2017;20(2):92-8. https://www.asep.org/asep/asep/JEPonlineAPRIL2017_Suksom.pdf

27. Porcari JP, Probst L, Forrester K, Doberstein S, Foster C, Cress ML, et al. Effect of wearing the elevation training mask on aerobic capacity, lung function, and hematological variables. J Sport Sci Med. 2016;15(2):379-86. https://www.ncbi.nlm.nih.gov/pmc/articles/PMC4879455/

28. Brocherie F, Girard O, Faiss R, Millet GP. High-intensity intermittent training in hypoxia: A double-blinded, placebo-controlled field study in youth football players. J Strength Cond Res. 2015;29(1):226-37. DOI: https://doi.org/10.1519/JSG.0000000000000590 
29. Buchheit M, Kuitunen S, Voss SC, Williams BK, Mendez-Villanueva A, Bourdon APG. Physiological Strain Associated With High- Intensity Hypoxic Intervals In Highly Trained Young Runners. J Strength Cond Res. 2012;26(1):94-105. https://doi.org/10.1519/ JSG.0b013e3182184fcb

30. Czuba M, ZająC A, Maszczyk A, Roczniok R, Poprzęcki S, Garbaciak W, et al. The effects of high intensity interval training in normobaric hypoxia on aerobic capacity in basketball players. J Hum Kinet. 2013;39(1):103-14. https://doi.org/10.2478/hukin-2013-0073

31. Robertson EY, Saunders PU, Pyne DB, Aughey RJ, Anson JM, Gore CJ. Reproducibility of performance changes to simulated live high/train low altitude. Med Sci Sports Exerc. 2010;42(2):394-401. DOI: https://doi.org/10.1249/MSS.0b013e3181b34b57

32. Siebenmann C, Robach P, Jacobs RA, Rasmussen P, Nordsborg N, Diaz V, et al. Live high-train low using normobaric hypoxia: A double-blinded, placebo-controlled study.J Appl Physiol. 2012;112(1):106-17. DOI: https://doi.org/10.1152/japplphysiol.00388.2011

33. McLean BD, Gore CJ, Kemp J. Application of 'Live Low-Train High' for Enhancing Normoxic Exercise Performance in Team Sport Athletes. Sport Med. 2014;44(9):127587. DOI: https://doi.org/10.1007/s40279-014-0204-8

34. Robach P, Hansen J, Pichon A, Meinild Lundby A-K, Dandanell S, Slettaløkken Falch $\mathrm{G}$, et al. Hypobaric live high-train low does not improve aerobic performance more than live low-train low in cross-country skiers. Scand J Med Sci Sports [Internet]. 2018 Jun 1;28(6):1636-52. DOI: https://doi.org/10.1111/sms.13075

35. Vogt, M. Hoppeler H. Is hypoxia training good for muscles and exercise performance? Prog Cardiovasc Dis. 2010;52(6):525-533. https://doi.org/10.1016/j.pcad.2010.02.013

36. Imray C, Booth A, Wright A, Bradwell A. Acute altitude illnesses. BMJ. 201 1;343:d4943.

37. Imray C, Wright A, Subudhi A, Roach R. Acute mountain sickness: Pathophysiology, prevention, and treatment. Prog Cardiovasc Dis. 2010;52(6):467-84. DOI: https://doi. org/10.1016/j.pcad.2010.02.003

38. Navarrete-Opazo A, Mitchell GS. Therapeutic potential of intermittent hypoxia: A matter of dose. Am J Physiol - Regul Integr Comp Physiol. 2014;307(10):1181-97. DOI: https://doi.org/10.1152/ajpregu.00208.2014

39. Lizamore CA, Hamlin MJ. The Use of Simulated Altitude Techniques for Beneficial Cardiovascular Health Outcomes in Nonathletic, Sedentary, and Clinical Populations: A Literature Review. High Alt Med Biol. 2017;18(4):305-21. https://doi.org/10.1089/ ham.2017.0050

40. Millet GP, Debevec T, Brocherie F, Malatesta D, Girard O. Therapeutic use of exercising in hypoxia: Promises and limitations. Front Physiol. 2016;7(JUN):2014-7. DOI: https:// doi.org/10.3389/fphys.2016.00224

41. Wilber RL. Current trends in altitude training Sports Medicine. Sport Med. 2001;31(4):249-65. DOI: https://doi.org/10.2165/00007256-200131040-00002 
42. Balsalobre-Fernández C, Tejero-González C, Campo-Vecino J, Alonso-Curiel D. La exposición hipóxica como medio para aumentar el rendimiento deportivo: ¿mito o realidad? Rev Int Med y Ciencias la Act Fis y del Deport. 2014;14(53):12-6. https://revistas. uam.es/rimcafd/article/view/3925

43. Foster C, Farl CV., Guidotti F, Harbin M, Roberts B, Schuette J, et al. The effects of high intensity interval training vs steady state training on aerobic and anaerobic capacity. J Sport Sci Med. 2015;14(4):747-55. https://www.ncbi.nlm.nih.gov/pmc/articles/ PMC4657417/

44. Jelleyman C, Yates T, O’Donovan G, Gray LJ, King JA, Khunti K, et al. The effects of high-intensity interval training on glucose regulation and insulin resistance: A meta-analysis. Obes Rev. 2015;16(11):942-61. DOI: https://doi.org/10.1111/obr.12317

45. Cofré-Bolados C, Sánchez-Aguilera P, Zafra-Santos E, Espinoza-Salinas A. Entrenamiento aeróbico de alta intensidad: Historia y fisiología clínica del ejercicio. Rev la Univ Ind Santander Salud. 2016;48(3):275-84. DOI: http://dx.doi.org/10.18273/revsal. v48n3-2016001

46. Gibala MJ, Gillen JB, Percival ME. Physiological and Health-Related Adaptations to Low-Volume Interval Training: Influences of Nutrition and Sex. Sport Med. 2014;44:12737. DOI: https://doi.org/10.1007/s40279-014-0259-6

47. MacInnis MJ, Gibala MJ. Physiological adaptations to interval training and the role of exercise intensity. J Physiol. 2017;595(9):2915-30. DOI: https://doi.org/10.1113/ JP273196

48. Atakan MM, Güzel Y, Bulut S, Koşar Nş, Mcconell GK, Turnagöl HH. Six HIIT sessions over 5 days increases VO2max, endurance capacity and sub-maximal exercise fat oxidation as much as 6 high-intensity interval training sessions over 2 weeks. J Sport Heal Sci. 2020;9(3):0-27. DOI: https://doi.org/10.1016/j.jshs.2020.06.008

49. Holloszy JO, Coyle EF. Adaptations of skeletal muscle to endurance exercise and their metabolic consequences. J Appl Physiol. 1984;121(6):831-8. DOI: https://doi. org/10.1152/jappl.1984.56.4.831

50. Coffey VG, Hawley JA. The molecular bases of training adaptation. Sport Med. 2007;37(9):737-63. DOI: https://doi.org/10.2165/00007256-200737090-00001

51. Montero D, Cathomen A, Jacobs RA, Flück D, de Leur J, Keiser S, et al. Haematological rather than skeletal muscle adaptations contribute to the increase in peak oxygen uptake induced by moderate endurance training. J Physiol. 2015;593(20):4677-88. DOI: https://doi.org/10.1113/JP270250

52. Bandeira E, Lopes-Pacheco M, Chiaramoni N, Ferreira D, Fernandez-Ruocco MJ, Prieto $\mathrm{MJ}$, et al. Association with amino acids does not enhance efficacy of polymerized liposomes as a system for lung gene delivery. Front Physiol. 2016;7(APR):1-9. DOI: https:// doi.org/10.3389/fphys.2016.00151

53. Goodman JM, Liu PP, Green HJ. Left ventricular adaptations following short-term endurance training. J Appl Physiol. 2005;98(2):454-60. DOI: https://doi.org/10.1152/japplphysiol.00258.2004 
54. NÍ Chéilleachair NJ, Harrison AJ, Warrington GD. HIIT enhances endurance performance and aerobic characteristics more than high-volume training in trained rowers. J Sport Sci. 2017;35(11):1052-8. DOI: https://doi.org/10.1080/02640414.2016.1209539

55. Bruseghini P, Calabria E, Tam E, Milanese C, Oliboni E, Pezzato A, et al. Effects of eight weeks of aerobic interval training and of isoinertial resistance training on risk factors of cardiometabolic diseases and exercise capacity in healthy elderly subjects. Oncotarget. 2015;6(19):16998-7015. DOI: https://doi.org/10.18632/oncotarget.4031

56. Safiyari-Hafizi H, Taunton J, Ignaszewski A, Warburton DER. The Health Benefits of a 12-Week Home-Based Interval Training Cardiac Rehabilitation Program in Patients With Heart Failure. Can J Cardiol. 2016;32(4):561-7. DOI: https://doi.org/10.1016/j. cjca.2016.01.031

57. Kong Z, Fan X, Sun S, Song L, Shi Q, Nie J. Comparison of high-intensity interval training and moderate-to-vigorous continuous training for cardiometabolic health and exercise enjoyment in obese young women: A randomized controlled trial. PLoS ONE. 2016;11(7):1-16. DOI: https://doi.org/10.1371/journal.pone.0158589

58. Klausen K, Robinson S, Micahel ED, Myhre LG. Effect of high altitude on maximal working capacity. J Appl Physiol. 1966;21(4):1191-4. DOI: https://doi.org/10.1152/jappl.1966.21.4.1191

59. Weil J V., Jamieson G, Brown DW, Grover RF. The red cell mass--arterial oxygen relationship in normal man. Application to patients with chronic obstructive airway disease. J Clin Invest. 1968;47(7):1627-39. DOI: https://doi.org/10.1172/JCI105854

60. Levine BD, Stray-Gundersen J. A practical approach to altitude training: Where to live and train for optimal performance enhancement. Int J Sports Med. 1992;13(SUPPL. 1). DOI: https://doi.org/10.1055/s-2007-1024642

61. Bailey DM. Physiological implications of altitude training for endurance performance at sea level: A review. Br J Sports Med. 1997;31(3):183-90. DOI: http://dx.doi. org/10.1136/bjsm.31.3.183

62. Jackson CGR, Sharkey BJ. Altitude, Training and Human Performance. Sport Med. 1988;6(5):279-84. DOI: https://doi.org/10.2165/00007256-198806050-00003

63. Hickson RC, Bomze HA, Holloszy JO. Linear increase in aerobic power induced by a strenuous program of endurance exercise. Journal of Applied Physiology. 1977;42(3):372-6. DOI: https://doi.org/10.1152/jappl.1977.42.3.372

64. Man Maria C, Ganera C. A study on the influence of training at altitude (2000m) on the blood hemoglobin and erythroietin values in athletics (aerobic resistance). Sci Mov Heal. 2015;15(2):409-19.

65. Czuba M, Fidos-Czuba O, Płoszczyca K, Zajac A, Langfort J. Comparison of the effect of intermittent hypoxic training vs. the live high, train low strategy on aerobic capacity and sports performance in cyclists in normoxia. Biol Sport. 2018;35(1):39-48. DOI: https://doi.org/10.5114/biolsport.2018.70750 
66. Czuba M, Bril G, Płoszczyca K, Piotrowicz Z, Chalimoniuk M, Roczniok R, et al. Intermittent hypoxic training at lactate threshold intensity improves aiming performance in well-trained biathletes with little change of cardiovascular variables. Biomed Res Int. 2019;2019:1287506. DOI: https://doi.org/10.1155/2019/1287506

67. Álvarez-Herms J, Julià-Sánchez S, Corbi F, Pagès T, Viscor G. A program of circuit resistance training under hypobaric hypoxia conditions improves the anaerobic performance of athletes. Sci Sport. 2016;31(2):78-87. DOI: https://doi.org/10.1016/j.scispo.2015.08.005

68. Park HY, Shin C, Lim K. Intermittent hypoxic training for 6 weeks in 3000 m hypobaric hypoxia conditions enhances exercise economy and aerobic exercise performance in moderately trained swimmers. Biol Sport. 2018;35(1):49-56. DOI: https://doi. org/10.5114/biolsport.2018.70751 